\title{
Association of Baseline Smoking Status with Long-Term Prognosis in Patients Who Underwent Percutaneous Coronary Intervention: Large Single-Center Data
}

\author{
Ru Liu (D), Zhan Gao, Huanhuan Wang, Xiaofang Tang, Lijian Gao, Ying Song, Jingjing Xu, \\ Jue Chen, Shubin Qiao, Yuejin Yang, Runlin Gao, Bo Xu $\mathbb{D}$, and Jinqing Yuan \\ Department of Cardiology, Fuwai Hospital, Chinese Academy of Medical Sciences and Peking Union Medical College, Beijing, China \\ Correspondence should be addressed to Bo Xu; bxu@citmd.com and Jinqing Yuan; dr_jinqingyuan@sina.com
}

Received 20 January 2019; Revised 10 March 2019; Accepted 15 April 2019; Published 5 May 2019

Academic Editor: Thach N. Nguyen

Copyright (C) $2019 \mathrm{Ru}$ Liu et al. This is an open access article distributed under the Creative Commons Attribution License, which permits unrestricted use, distribution, and reproduction in any medium, provided the original work is properly cited.

\begin{abstract}
Objectives. This study analyzed a large sample to explain the association of baseline smoking state with long-term prognosis of coronary artery disease (CAD) patients who underwent percutaneous coronary intervention (PCI). Background. Data is limited up to now regarding whether smoker's paradox exists in Chinese population. Methods. A total of 10724 consecutive cases were enrolled from January to December 2013. 2-year clinical outcomes were evaluated among current smokers and nonsmokers. Major adverse coronary event (MACCE) included all-cause death, revascularization, myocardial infarction (MI), and stroke. Results. Current smokers and nonsmokers accounted for $57.1 \%$ and $42.9 \%$, respectively. Current smokers were presented with predominant male sex, lower age, and less comorbidities. The rates of 2-year all-cause death were not significantly different among two groups. But the rate of stroke and bleeding was significantly higher in nonsmokers than in current smokers $(1.6 \%$ and $1.1 \%, P=0.031 ; 7.2 \%$ and $6.1 \%$, $P=0.019)$. The rate of revascularization was significantly higher in current smokers than in nonsmokers $(9.1 \%$ and $8.0 \%, P=0.037)$. Multivariable Cox regression indicated that, compared with nonsmokers, current smokers were not independently associated with all endpoints (all $P>0.05$ ). Conclusions. 2-year all-cause death, MACCE, MI, revascularization, stroke, ST, and bleeding risk were similar between current smokers and nonsmokers in CAD patients undergoing PCI.
\end{abstract}

\section{Introduction}

Cigarette smoking is generally known as an important risk factor for pathogenesis of coronary artery disease (CAD), as well as prognosis [1-8]. Smoking cessation is recommended by international guidelines as one of crucial measurements for secondary prevention regardless of revascularization [912]. However, several studies have demonstrated a higher incidence of acute myocardial infarction (MI) but improved or neutral outcome after reperfusion among smokers than nonsmokers, which is termed the smoker's paradox [13-17]. Several small-sample cohort studies in Chinese CAD patients who underwent percutaneous coronary intervention (PCI) reported more nonfatal MI, but similar all-cause death risk in smokers than nonsmokers [18-20]. Therefore, it is uncertain whether the phenomenon of the smoker's paradox exists in Chinese population. Large data is limited up to now. This problem is of paramount importance for secondary prevention management for CAD patients. This study analyzed a large single-center sample in China to explain the impact of smoking state at baseline on long-term prognosis of CAD patients who received PCI.

\section{Methods}

2.1. Ethical Statement. Ethical approvals were obtained from the Fuwai Hospital Research Ethics Committees (No. 2013449). The Institutional Review Board approved the study protocol and all patients signed written informed consent before the intervention, including full set of risk-informed consent and information use consent for scientific purposes.

2.2. Study Population. A total of 10724 consecutive cases with CAD who underwent PCI were enrolled from January to 
December 2013 in our center, the largest cardiovascular center of China. Smoking state at baseline was defined according to smoking history recorded at admission. Nonsmokers included patients who never smoked and those former smokers without cigarette usage in recent 3 months. The rest was defined as current smokers regardless of reduced quantity of smoking or quitting cigarette less than 3 months. Diagnosis of ST-segment elevated myocardial infarction (STEMI), non-ST-segment elevated myocardial infarction (NSTEMI), unstable angina pectoris (UAP), and stable coronary artery disease (SCAD) was in terms of criteria based on the "2013 European Society of Cardiology (ESC) guidelines on the management of SCAD," "2015 ESC guidelines for the management of acute coronary syndromes (ACS) in patients presenting without persistent ST-segment elevation," and "fourth universal definition of myocardial infarction (2018)" $[9,10,21]$.

2.3. Procedural Details. Before selective PCI, if not taking long-term aspirin and clopidogrel, patients received 300 mg aspirin and P2Y12 inhibitor with loading dose orally. Patients with ACS scheduled for primary PCI received the same dose of aspirin and clopidogrel (loading dose $300 \mathrm{mg}$ or $600 \mathrm{mg}$, according to bleeding risk) as soon as possible. Ticagrelor was seldom used in our center in the year of 2013, only when clopidogrel resistance was observed and patients were willing to take it on their own expense (loading dose of $180 \mathrm{mg}$ or cumulative dose of $180 \mathrm{mg}$ followed by $90 \mathrm{mg}$ twice a day). Before coronary angiography (CAG), $3000 \mathrm{U}$ heparin sodium was administered through an arterial sheath or intravenously. Before PCI, $100 \mathrm{U} / \mathrm{kg}$ of heparin sodium was administered. The dose was lowered to $50-70 \mathrm{U} / \mathrm{kg}$ in patients over the age of seventy to reduce bleeding risk. If PCI proceeded for more than $1 \mathrm{~h}$, an additional $1000 \mathrm{U}$ of heparin sodium was administered. Results of CAG were read by experienced cardiologists. More than 50\% stenosis of left main artery (LM), left anterior descending artery (LAD), left circumflex artery (LCX), right coronary artery (RCA), and main branch of these vessels was defined as coronary artery stenosis. More than $70 \%$ stenosis of the vessels mentioned above, along with ischemic symptoms or ischemic evidence shown by examinations, was indicated for coronary stent implantation. Three-vessel disease (TVD) was defined as angiographic stenosis of $\geq 50 \%$ in all three main coronary arteries, LAD, LCX, and RCA. Synergy between Percutaneous Coronary Intervention with Taxus and Cardiac Surgery (SYNTAX) score (SS) and residual SNYTAX score (rSS) was assessed by two of the three experienced cardiologists in an independent angiographic core laboratory, who were blinded to clinical outcomes. High, intermediate, and low SS were defined as $\mathrm{SS} \geq 33,23 \leq \mathrm{SS}<33$, and $0 \leq \mathrm{SS}<23$. Incomplete revascularization (ICR) was defined as $\mathrm{rSS} \geq 8$, which was identified as a level strongly associated with increased cardiac death, MI, revascularization, and MACCE [22].

2.4. Follow-Up and Definitions. The patients were visited 30 days and 6 months after PCI and every 1 year thereafter. Information of in-hospital outcome was obtained through review of medical records, and the long-term clinical outcome was collected from survey completed by telephone follow-up, follow-up letter, or visit. A group of independent clinical physicians oversaw checking and confirmed all adverse events carefully. Investigators training, blinded questionnaire filling, and telephone recording were performed to control the data quality.

Primary endpoint was all-cause death. Composite endpoint was defined as major adverse coronary events (MACCEs), including all-cause death, revascularization, $\mathrm{MI}$, and stroke. Secondary endpoints were MACCE, cardiac death, revascularization, MI, stroke, stent thrombosis (ST), and bleeding. Cardiac death is identified as death caused by MI, heart failure, and/or malignant arrhythmia definitely, or death which cannot be explained clearly by other reasons. ST was defined as definite, probable, and possible ST based on the Academic Research Consortium criteria. Bleeding was defined according to criteria established by Bleeding Academic Research Consortium (BARC), excluding BARC 0 and 1 types.

2.5. Statistical Analysis. Data statistics was applied using SPSS 22.0 (IBM Corp., Armonk, New York, USA). Student's t-tests were used to compare continuous variables while Chisquare tests were applied to compare categorical variables between the two groups. Kaplan-Meier curves were drawn to compare cumulative event rates of the two groups. Multivariate Cox proportional hazard regression analyses were applied to control baseline confounders. Covariates for Cox regression were those variables with significant differences in baseline or important clinical meaning. All $\mathrm{P}$ values were two sided with a significance level of 0.05 . Tendency of significant difference was judged when $0.05<\mathrm{P}<0.1$.

\section{Results}

3.1. Baseline Characteristics. Among 10724 cases analyzed, current smokers and nonsmokers accounted for $57.1 \%$ and $42.9 \%$, respectively. In current smokers, majority (96.0\%) were male, which was significantly higher than that in nonsmokers $(52.0 \%, P<0.001)$. The average age of current smokers was 4.2 years younger than that of nonsmokers. Current smokers had significantly higher BMI than nonsmokers $(26.1 \pm 3.2$ and $25.8 \pm 3.2, P<0.001)$. Current smokers had less comorbidities of hypertension (61.9\% and $67.7 \%$, $P<0.001)$ and diabetes mellitus (DM) $(29.2 \%$ and $31.6 \%$, $P=0.008)$, but more family history of CAD $(26.4 \%$ and $22.5 \%, P<0.001)$, previous $\mathrm{MI}(22.1 \%$ and $15.3 \%, P<0.001)$, and prior PCI or CABG $(28.1 \%$ and $23.7 \%, P<0.001)$ than nonsmokers. Current smokers had lower LVEF level (62.3 \pm 7.4 and $63.3 \pm 7.1, P<0.001)$, but higher eGFR $(93.0 \pm$ 14.7 and $89.0 \pm 15.4, P<0.001)$ than nonsmokers. Angina pectoris happened less $(70.6 \%$ and $78.5 \%, P<0.001)$, while STEMI occurred more, $(16.2 \%$ and $10.0 \%, P<0.001)$ in current smokers than nonsmokers. Antiplatelet drugs and statin were similarly applied between 2 groups $(P>0.05)$, while $\beta$-blocker and calcium antagonist were less prescribed at discharge in current smokers than nonsmokers $(89.6 \%$ and $91.0 \%$, $P=0.018 ; 46.4 \%$ and $51.6 \%, P<0.001$ ). (Table 1 ) 
TABLE 1: The baseline clinical, angiographic, and procedural characteristics and medication situation.

\begin{tabular}{|c|c|c|c|c|}
\hline Variables & $\begin{array}{c}\text { All } \\
(\mathrm{n}=10724)\end{array}$ & $\begin{array}{l}\text { Current } \\
\text { smokers } \\
(\mathrm{n}=6123)\end{array}$ & $\begin{array}{l}\text { Nonsmokers } \\
\quad(\mathrm{n}=4601)\end{array}$ & $P$ value \\
\hline \multicolumn{5}{|l|}{ Demographic characteristics } \\
\hline Male gender, $\%$ & $8272(77.1)$ & $5880(96.0)$ & $2392(52.0)$ & $<0.001$ \\
\hline Age, years & $58.4 \pm 10.3$ & $56.6 \pm 10.0$ & $60.8 \pm 10.2$ & $<0.001$ \\
\hline BMI, kg/m2 & $25.9 \pm 3.2$ & $26.1 \pm 3.2$ & $25.8 \pm 3.2$ & $<0.001$ \\
\hline \multicolumn{5}{|l|}{ Coexisting conditions, $\%$} \\
\hline Hypertension & $6906(64.4)$ & $3789(61.9)$ & $3117(67.7)$ & $<0.001$ \\
\hline $\mathrm{DM}$ & $3238(30.2)$ & $1786(29.2)$ & $1452(31.6)$ & 0.008 \\
\hline Hyperlipidemia & $7211(67.2)$ & $4138(67.6)$ & $3073(66.8)$ & 0.387 \\
\hline Previous MI & $2061(19.2)$ & $1355(22.1)$ & $706(15.3)$ & $<0.001$ \\
\hline Prior PCI or CABG & $2808(26.2)$ & $1719(28.1)$ & $1089(23.7)$ & $<0.001$ \\
\hline Family history of CAD & $2651(24.7)$ & $1614(26.4)$ & $1037(22.5)$ & $<0.001$ \\
\hline CVD & $1150(10.7)$ & $643(10.5)$ & $507(11.0)$ & 0.391 \\
\hline PVD & $288(2.7)$ & $177(2.9)$ & $111(2.4)$ & 0.129 \\
\hline COPD & $247(2.3)$ & $143(2.3)$ & $104(2.3)$ & 0.799 \\
\hline $\operatorname{LVEF}(\%)$ & $62.8 \pm 7.4$ & $62.3 \pm 7.4$ & $63.3 \pm 7.1$ & $<0.001$ \\
\hline \multicolumn{5}{|l|}{ Clinical presentation, $\%$} \\
\hline Asymptomatic ischemia & $869(8.1)$ & $523(8.5)$ & $346(7.5)$ & $<0.001$ \\
\hline Stable angina & $3424(31.9)$ & $1842(30.1)$ & $1582(34.4)$ & $<0.001$ \\
\hline Unstable angina pectoris & $4509(42.0)$ & $2479(40.5)$ & $2030(44.1)$ & $<0.001$ \\
\hline AMI & $1922(17.9)$ & $1279(20.9)$ & $643(14.0)$ & $<0.001$ \\
\hline STEMI & $1447(13.5)$ & $989(16.2)$ & $458(10.0)$ & $<0.001$ \\
\hline NSTEMI & $475(4.4)$ & $290(4.7)$ & $185(4.0)$ & 0.075 \\
\hline \multicolumn{5}{|l|}{ Laboratory examination } \\
\hline eGFR before PCI, $\mathrm{mL} / \mathrm{min} / 1.73 \mathrm{~m} 2$ & $91.3 \pm 15.1$ & $93.0 \pm 14.7$ & $89.0 \pm 15.4$ & $<0.001$ \\
\hline HGB before PCI, g/L & $141.0 \pm 15.8$ & $144.8 \pm 14.4$ & $136.1 \pm 16.2$ & $<0.001$ \\
\hline PLT before PCI, $10^{9} / \mathrm{L}$ & $203.6 \pm 54.4$ & $201.4 \pm 53.6$ & $206.4 \pm 55.4$ & $<0.001$ \\
\hline Urine acid, $\mu \mathrm{mol} / \mathrm{L}$ & $341.6 \pm 84.7$ & $353.2 \pm 82.1$ & $326.2 \pm 85.7$ & $<0.001$ \\
\hline HbAlc, \% & $6.6 \pm 1.2$ & $6.6 \pm 1.2$ & $6.6 \pm 1.3$ & 0.029 \\
\hline $\mathrm{LDL}-\mathrm{C}, \mathrm{mmol} / \mathrm{L}$ & $2.50 \pm 0.90$ & $2.48 \pm 0.88$ & $2.54 \pm 0.93$ & $<0.001$ \\
\hline $\mathrm{ESR}, \mathrm{mm} / \mathrm{h}$ & $10.8 \pm 11.3$ & $9.3 \pm 10.2$ & $12.9 \pm 12.3$ & $<0.001$ \\
\hline \multicolumn{5}{|l|}{ Angiographic and procedural characteristics } \\
\hline SNYTAX score & $11.7 \pm 8.1$ & $11.5 \pm 8.1$ & $11.9 \pm 8.1$ & 0.019 \\
\hline Residual SNYTAX & $3.4 \pm 5.7$ & $3.4 \pm 5.6$ & $3.5 \pm 5.8$ & 0.334 \\
\hline LM or TVD, $\%$ & $457(4.3)$ & $267(4.4)$ & $190(4.1)$ & 0.558 \\
\hline LAD involved, $\%$ & $9702(90.5 \%)$ & $5480(89.5)$ & $4222(91.8)$ & $<0.001$ \\
\hline No. of target lesions & $1.40 \pm 0.66$ & $1.41 \pm 0.67$ & $1.39 \pm 0.65$ & 0.098 \\
\hline No. of stents per patient & $1.80 \pm 1.11$ & $1.82 \pm 1.13$ & $1.79 \pm 1.08$ & 0.197 \\
\hline Time of procedure, min & $36.7 \pm 31.5$ & $37.4 \pm 33.6$ & $35.6 \pm 28.5$ & 0.002 \\
\hline Procedure and stent type, \% & & & & 0.019 \\
\hline PTCA & $237(2.2)$ & $121(2.0)$ & $116(2.5)$ & \\
\hline BMS & $64(0.6)$ & $30(0.5)$ & $34(0.7)$ & \\
\hline First-generation durable polymer DES & $597(5.6)$ & $363(5.9)$ & $234(5.1)$ & \\
\hline Second-generation durable polymer DES & $6094(56.8)$ & $3428(56.0)$ & $2666(57.9)$ & \\
\hline Domestic biodegradable polymer DES & $1572(14.7)$ & $925(15.1)$ & $647(14.1)$ & \\
\hline Mixed implantation of DES & $1692(15.8)$ & $976(15.9)$ & $716(15.6)$ & \\
\hline Others (Janus, Yinyi) & $167(1.6)$ & $93(1.5)$ & $74(1.6)$ & \\
\hline Procedure unsuccess & $301(2.8)$ & $187(3.1)$ & $114(2.5)$ & \\
\hline
\end{tabular}


TABLE 1: Continued.

\begin{tabular}{|c|c|c|c|c|}
\hline Variables & $\begin{array}{c}\text { All } \\
(\mathrm{n}=10724)\end{array}$ & $\begin{array}{l}\text { Current } \\
\text { smokers } \\
(\mathrm{n}=6123)\end{array}$ & $\begin{array}{c}\text { Nonsmokers } \\
\quad(\mathrm{n}=4601)\end{array}$ & $P$ value \\
\hline \multicolumn{5}{|c|}{ Medication at discharge, $\%$} \\
\hline Aspirin & $10585(98.7)$ & $6047(98.8)$ & $4538(98.6)$ & 0.562 \\
\hline Clopidogrel & $10701(99.8)$ & $6114(99.9)$ & 4587 (99.7) & 0.081 \\
\hline Ticagrelor & $19(0.2)$ & $9(0.1)$ & $10(0.2)$ & 0.391 \\
\hline DAPT & $10583(98.7)$ & $6047(98.8)$ & $4536(98.6)$ & 0.440 \\
\hline Statin & $10285(95.9)$ & $5878(96.0)$ & 4407 (95.8) & 0.578 \\
\hline Calcium antagonist & $5216(48.6)$ & $2842(46.4)$ & $2374(51.6)$ & $<0.001$ \\
\hline$\beta$-blocker & $9673(90.2)$ & $5487(89.6)$ & $4186(91.0)$ & 0.018 \\
\hline
\end{tabular}

AMI: acute myocardial infarction; BMI: body mass index; BMS: bare metal stent; CAD: coronary artery disease; CABG: coronary artery bypass grafting; COPD: chronic obstructive pulmonary disease; CVD: cerebral vascular disease; DES: drug-eluting stent; DM: diabetes mellitus; DAPT: dual antiplatelet therapy; eGFR: estimated glomerular filtration rate; ESR: erythrocyte sedimentation rate; HGB: hemoglobin; HbAlc: hemoglobin Alc; LAD: left anterior descending artery; LM: left main; LDL-C: low density lipoprotein cholesterol; LVEF: left ventricular ejection fraction; MI: myocardial infarction; NSTEMI: nonST-segment elevated myocardial infarction; PCI: percutaneous coronary intervention; PLT: platelet; PTCA: percutaneous transluminal coronary angioplasty; PVD: peripheral vascular disease; STEMI: ST-segment elevated myocardial infarction; SYNTAX: Synergy between Percutaneous Coronary Intervention with Taxus and Cardiac Surgery; TC: total cholesterol; TVD: three-vessel disease.

Data are expressed as mean \pm standard deviation, or counts (percentage).

TABLE 2: 2-year outcomes.

\begin{tabular}{|c|c|c|c|c|}
\hline Endpoints & $\begin{array}{c}\text { All } \\
(\mathrm{n}=10724)\end{array}$ & Current smokers $(\mathrm{n}=6123)$ & $\begin{array}{c}\text { Nonsmokers } \\
(\mathrm{n}=4601)\end{array}$ & $P$ value \\
\hline All-cause death & $131(1.2)$ & $70(1.1)$ & $61(1.3)$ & 0.394 \\
\hline MACCE & $1295(12.1)$ & $757(12.4)$ & $538(11.7)$ & 0.292 \\
\hline Cardiac death & $74(0.7)$ & $39(0.6)$ & $35(0.8)$ & 0.444 \\
\hline Myocardial infarction & $212(2.0)$ & $122(2.0)$ & $90(2.0)$ & 0.893 \\
\hline Revascularization & $923(8.6)$ & $557(9.1)$ & $366(8.0)$ & 0.037 \\
\hline Stent thrombosis & $91(0.8)$ & $53(0.9)$ & $38(0.8)$ & 0.825 \\
\hline Stroke & $145(1.4)$ & $70(1.1)$ & $75(1.6)$ & 0.031 \\
\hline Bleeding & $702(6.5)$ & $371(6.1)$ & $331(7.2)$ & 0.019 \\
\hline
\end{tabular}

Current smokers were implanted with the secondgeneration drug-eluting stents (DES) less than nonsmokers (56.0\% and 57.9\%, $P=0.043$ ). Current smokers had lower SNYTAX score $(11.5 \pm 8.1$ and $11.9 \pm 8.1, P=0.019)$ and less LAD involved lesions ( $89.5 \%$ and $91.8 \%, P<0.001$ ), but longer times of procedure than nonsmokers $(37.4 \pm 33.6$ and $35.6 \pm$ 28.5, $P=0.002$ ). (Table 1)

3.2. Clinical Outcomes and Subgroup Analysis. Clinical follow-up was completed for 10665 patients (99.4\%) of 2 years. The average follow-up was 872.4 days. The occurrence of adverse cardiovascular events in each group is listed in Table 2. During 2-year follow-up, the rates of all-cause death, MACCE, cardiac death, MI, and ST were not significantly different between the two groups (all $P>0.05$ ). But the rate of stroke and bleeding was significantly higher in nonsmokers than in current smokers $(1.6 \%$ and $1.1 \%, P=0.031 ; 7.2 \%$ and $6.1 \%, P=0.019)$. The rate of revascularization was significantly higher in current smokers than in nonsmokers $(9.1 \%$ and $8.0 \% ; P=0.037)$. Kaplan-Meier curves revealed the same finding. (Figure 1)

Multivariable Cox regression analysis indicated that current smokers, compared with nonsmokers, were not independently associated with 2-year all-cause death (HR $1.03,95 \%$ CI $0.69-1.54, P=0.881$ ), MACCE (HR 0.98, 95\%CI 0.86-1.12, $P=0.766$ ), and other all endpoints (all $P>0.05$ ). (Table 3)

Subgroups included male or female subgroups, patients with age $\geq 75$ or $<75$, patients diagnosed with STEMI, NSTEMI, UAP, or SCAD, patients with TVD or LM involved, patients with high, intermediate, or low SS, and patients who underwent CR or ICR. The interaction analysis showed classification of CAD was not independent of baseline smoking status $(P=0.038)$. COX regressions analysis showed that, in all subgroups except for TVD subgroup, current smokers, compared with nonsmokers, were also not independently associated with MACCE (all $P>0.05$ ). In patients with TVD, current smokers were associated with significantly higher MACCE risk compared with nonsmokers (HR 4.34, 95\%CI $1.19-15.85, P=0.026$ ). (Figure 2)

\section{Discussion}

Due, in part, to the prothrombotic effects of smoking, cigarette smokers are more likely to present with STEMI. The "smoker's paradox" is discussed mainly in STEMI patients. 
TABLE 3: Multivariate Cox regression analysis in whole cohort, SCAD subgroup, and ACS subgroup.

\begin{tabular}{|c|c|c|c|c|c|c|}
\hline \multirow{2}{*}{ Endpoints } & \multicolumn{2}{|c|}{ Whole cohort } & \multicolumn{2}{|c|}{ SCAD subgroup } & \multicolumn{2}{|c|}{ ACS subgroup } \\
\hline & HR $(95 \% \mathrm{CI})$ & $P$ value & HR $(95 \% \mathrm{CI})$ & $P$ value & HR (95\%CI) & $\mathrm{P}$ value \\
\hline All-cause death & $1.03(0.69,1.54)$ & 0.881 & $0.81(0.41,1.60)$ & 0.537 & $1.12(0.68,1.85)$ & 0.662 \\
\hline MACCE & $0.98(0.86,1.12)$ & 0.766 & $0.84(0.68,1.03)$ & 0.089 & $1.09(0.93,1.29)$ & 0.298 \\
\hline Cardiac death & $1.16(0.68,1.98)$ & 0.587 & $0.42(0.14,1.30)$ & 0.133 & $1.72(0.91,3.28)$ & 0.098 \\
\hline Myocardial infarction & $0.90(0.65,1.25)$ & 0.547 & $0.77(0.46,1.29)$ & 0.321 & $1.00(0.65,1.52)$ & 0.98 \\
\hline Revascularization & $0.97(0.83,1.13)$ & 0.687 & $0.81(0.64,1.04)$ & 0.097 & $1.10(0.90,1.34)$ & 0.362 \\
\hline Stent thrombosis & $1.03(0.64,1.65)$ & 0.92 & $0.52(0.22,1.25)$ & 0.143 & $1.47(0.82,2.64)$ & 0.196 \\
\hline Stroke & $1.17(0.79,1.71)$ & 0.434 & $1.07(0.57,2.03)$ & 0.83 & $1.26(0.78,2.03)$ & 0.349 \\
\hline Bleeding & $0.90(0.75,1.08)$ & 0.273 & $0.96(0.73,1.27)$ & 0.763 & $0.85(0.66,1.08)$ & 0.183 \\
\hline
\end{tabular}

ACS: acute coronary syndrome; SCAD: stable coronary artery disease.
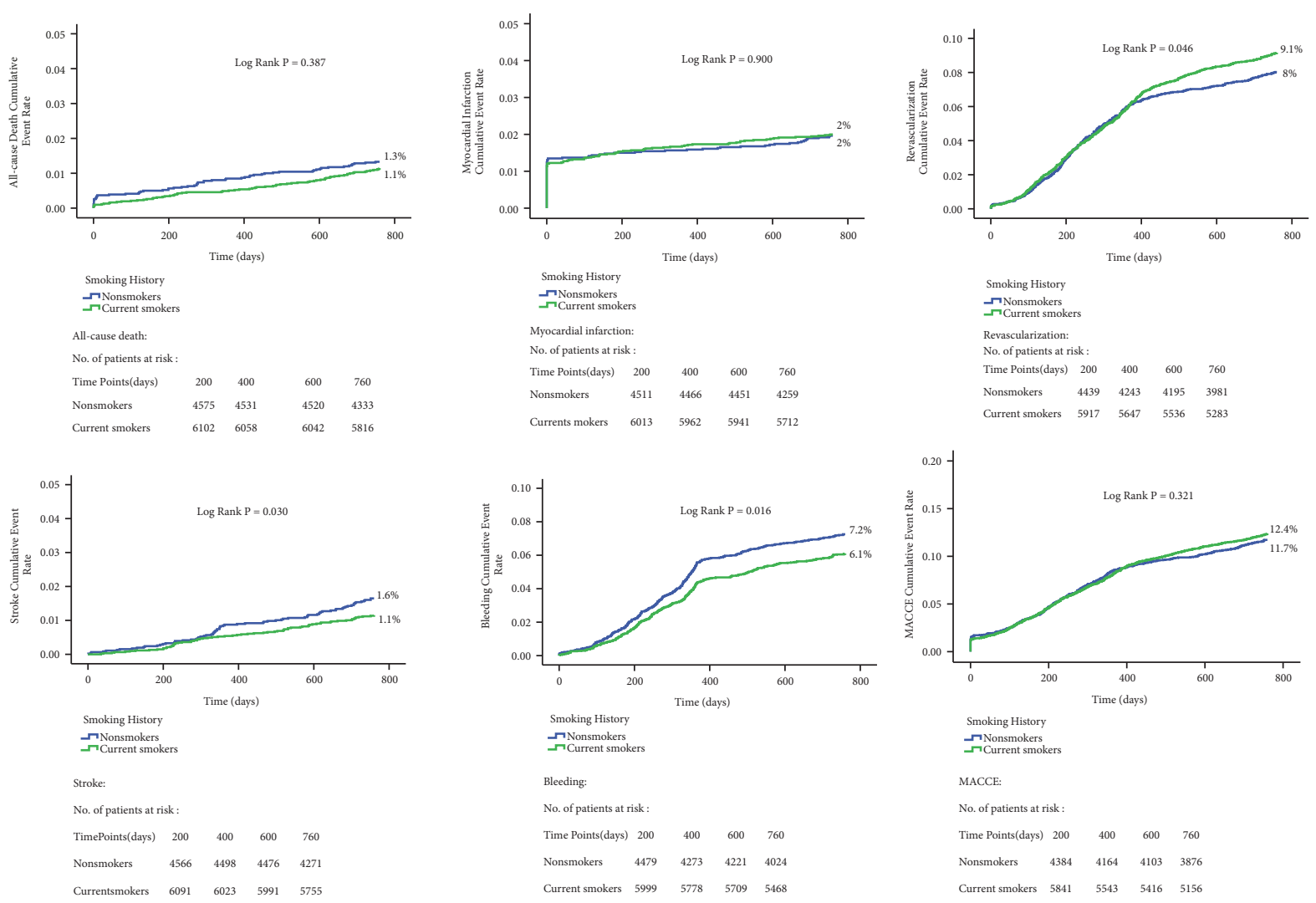

FIGURE 1: Kaplan-Meier survival curves between current smokers and nonsmokers.

The possible explanation is that smoking is associated with platelet aggregation and blood coagulability. Thus, coronary obstruction in smokers may be more thrombogenic and less atherosclerotic than in nonsmokers, leading more likely to be perfused spontaneously or by thrombolytic therapy [13$17,23-26]$.

In this study, we have shown in a large contemporary cohort of patients with CAD undergoing PCI that (1) 2year outcomes were similar between current smokers and nonsmokers; (2) MACCE risk was similar between current smokers and nonsmokers in all subgroups except for TVD subgroup. This finding indicated that the "smoker's paradox" also exists in CAD patients undergoing PCI in Chinese population. It is difficult to interpret that the results of impact of smoking on 2-year outcomes were not statistically significant. Possible explanation may focus on the damage mechanisms of cigarettes. The hypercoagulability of cigarette smokers may not only predispose them to the early occurrence of MI, but also could predispose them to reinfarction [15]. It was indeed observed in this study that smokers were associated with more STEMI and less angina pectoris than nonsmokers, and smoking status had significant interaction effect with classification of CAD. The hypercoagulability may be early inhibited by effective antithrombotic therapy. As the baseline 


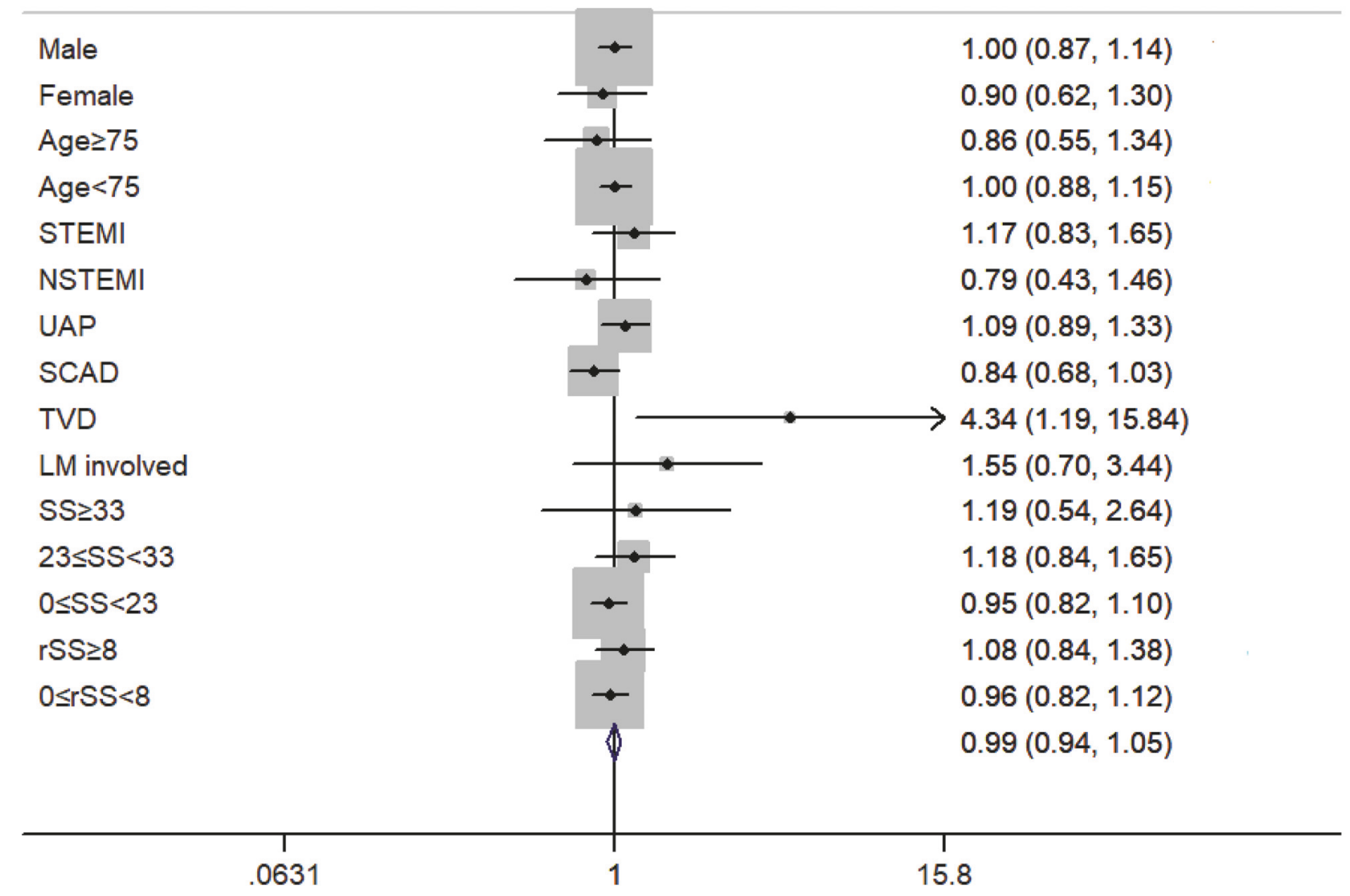

FIGURE 2: Forest plot of subgroup analysis. The multivariable analysis indicated whether current smoking is associated with 2-year MACCE.

medication analysis showed, antiplatelet drugs were highly prescribed in the two groups, showing no significant differences. Other pathological mechanisms shown in previous studies include an unfavorable modulation of autonomic cardiac control, leading to a shift towards sympathetic predominance accompanied by increased levels of catecholamines, lower arrhythmogenic threshold, increased vasoconstriction, and increased myocardial oxygen consumption [26-30]. The corresponding drug solutions involve appropriate $\beta$-blockers and calcium antagonists. The hazard of smoking may be offset by the comprehensive medication to some extent. Therefore, we think that this "neutral" result is inadequate to question the necessity of smoking cessation for secondary prevention. Cardiologists should continue to emphasize the pivotal role of smoking cessation in risk reduction especially in patients with established CAD.

On the other hand, both supporters and opponents of "smoker's paradox" found that smokers were associated with younger age, more male sex, and less comorbidities compared with nonsmokers. Some researchers indicated that the phenomenon of "smoker's paradox" could be partly explained by fewer coexisting high-risk features in patients with AMI who are currently smoking [13-17, 23-25, 31, 32]. In our study, the "lower risk" of stroke and bleeding in current smokers may be partly explained in a similar fashion to that seen in patients with AMI, that baseline characteristics do play important role in data interpretation. Current smokers were associated with younger age, predominant of male sex, less DM, and hypertension. All these factors may reduce stroke and bleeding risk and be considered as confounders.

The results also showed that current smokers were associated with more revascularization than nonsmokers in univariate analysis, but the correlation was no longer significant after multivariate adjustment. It means that the explanation lied in baseline differences. However, except for less second-generation durable polymer DES implantation in current smokers, baseline analysis showed better general conditions and less severe lesions of current smokers than nonsmokers, revealed by more male, younger age, less DM and hypertension, higher eGFR, lower SNYTAX scores, and less LAD involved lesions, which cannot answer why current smokers had increased revascularization rate. It is worth noting that current smokers had higher BMI, higher urine acid level, and more family history of CAD than nonsmokers. It is indicated that possibly genetic and metabolic mechanisms are involved in the relationship between smoking and revascularization after PCI. As known, smokers are always a group of people keeping ill habits. Genetic susceptibility and unhealthy way of life may explain current smokers' increased revascularization rate to some extent. Interestingly, current smokers also had more previous MI and prior PCI or CABG 
history. The repeated hospitalization and revascularization did not convert to mortality; however, they inevitably contributed to increased burden of governmental health fund.

Additionally, patients with LM involved or TVD were not different from current smokers and nonsmokers in this study, not consistent with previous data. However, in subgroup of patients with TVD, current smokers had about 4.3 times MACCE risk than nonsmokers. Because event rates of allcause death, MI, and stroke were low in TVD subgroup, COX regression models were not significant. We could not find out which endpoint current smoking really effects. As reported in the Chinese postmenopausal women, current smoking and the presence of multiple-vessel disease can independently predict events of all-cause death, nonfatal infarction, or unstable angina [33]. It is reasonable to speculate that smoking has effect on outcomes of patients who suffered from severe CAD.

In general, this study analyzed the association between baseline smoking status and 2-year outcomes in a largesample cohort with CAD who underwent PCI, expecting to find some adverse impacts of smoking as routing perception. Unexpectedly, the results were not statistically significant. How to interpret the results for clinical practice. Maybe we should be conscious of the complicated clinical situation that smoking possibly plays more important role in initial triggering mechanisms in ACS, due to vasoconstriction, hypercoagulability, platelet aggregation, and endothelial dysfunction [13-15, 23-30]. However, the progression of atherosclerosis can be influenced by multifactors, including genetic mechanisms and secondary medication $[34,35]$. Genetic factors were not included in most clinical studies in this area for uncertainty up to date. And, secondary medication may be of conversing effect of smoking to some extent. Antiplatelet and anticoagulation therapy may reverse the platelet aggregation and hypercoagulability driven by tobacco. $\beta$-blockers and calcium antagonists may decrease oxygen consumption and relieve vasoconstriction. And statins may protect endothelium and anti-inflammation. The considerate secondary medication for patients who receive PCI in our center may explain the results in this study to some extent. Further research is required to demonstrate the speculation.

Several limitations should be taken into consideration. Firstly, former smokers were much less than current smokers and never smokers, which made comparisons among current smokers, former smokers, and never smokers lack statistical power. Therefore, former smokers and never smokers were included into one group. The heterogeneity between former smokers and never smokers may affect results to some extent. Secondly, no data was available regarding the quantity or duration of smoking. Thus, we cannot analyze doseresponse or duration-response relationship between smoking and adverse events risk. Thirdly, no data was available on those who quit smoking after PCI, and how their event rate is compared to that of patients who continued to smoke. Finally, there may be additional confounders that are not controlled for within our model. Nevertheless, this is a large core laboratory analysis comparing current smokers and nonsmokers in patients with whole CAD spectrum in Chinese population, in terms of both outcomes and angiographic data, and we believe that we have accounted for the most clinically relevant variables in our model.

\section{Conclusions}

2-year all-cause death, MACCE, MI, revascularization, stroke, ST, and bleeding risk were all similar between current smokers and nonsmokers in CAD patients undergoing PCI. Subgroup analysis found that current smokers were associated with higher MACCE risk in patients with TVD.

\section{Data Availability}

The data used to support the findings of this study are available from the corresponding author upon request.

\section{Disclosure}

An earlier version of this work's abstract was presented in the Journal of the American College of Cardiology, 2018. None of the article contents are under consideration for publication in any other journal or have been published in any journal. All authors have participated in the work and have reviewed and agree with the content of the article.

\section{Conflicts of Interest}

We have no conflicts of interest to disclose.

\section{Authors' Contributions}

Ru Liu contributed to all aspects of this study, including study concept and design, data acquisition, statistical analysis and interpretation, and drafting and revising the report. Zhan Gao, Huanhuan Wang, Xiaofang Tang, Lijian Gao, Ying Song, Jingjing Xu, Jue Chen, Shubin Qiao, Yuejin Yang, and Runlin Gao contributed to data acquisition. Bo $\mathrm{Xu}$ and Jinqing Yuan contributed to initial study conception and design, data interpretation, and critical revision of the report. All authors have approved the final article.

\section{Acknowledgments}

This work is supported by the National Natural Science Foundation of China (Grants no. 81470486 and 81770365) and the National Key Research and Development Program of China during the 13th Five-Year Plan Period (Project no. 2016YFC1301301).

\section{References}

[1] J. A. Critchley and S. Capewell, "Mortality risk reduction associated with smoking cessation in patients with coronary heart disease: a systematic review," Journal of the American Medical Association, vol. 290, no. 1, pp. 86-97, 2003.

[2] P. Jha, C. Ramasundarahettige, V. Landsman et al., "21st-century hazards of smoking and benefits of cessation in the United 
States," The New England Journal of Medicine, vol. 368, no. 4, pp. 341-350, 2013.

[3] R. Sakata, P. McGale, E. J. Grant, K. Ozasa, R. Peto, and S. C. Darby, "Impact of smoking on mortality and life expectancy in Japanese smokers: A prospective cohort study," BMJ, vol. 345, no. 7883, 2012.

[4] J. A. Ambrose and R. S. Barua, "The pathophysiology of cigarette smoking and cardiovascular disease: an update," Journal of the American College of Cardiology, vol. 43, no. 10, pp. 1731-1737, 2004.

[5] M. Ezzati, S. J. Henley, M. J. Thun, and A. D. Lopez, "Role of smoking in global and regional cardiovascular mortality," Circulation, vol. 112, no. 4, pp. 489-497, 2005.

[6] M. J. Thun, B. D. Carter, D. Feskanich et al., "50-Year trends in smoking-related mortality in the United States," The New England Journal of Medicine, vol. 368, no. 4, pp. 351-364, 2013.

[7] V. Notara, D. B. Panagiotakos, S. Kouroupi et al., "Smoking determines the 10-year (2004-2014) prognosis in patients with Acute Coronary Syndrome: The GREECS observational study," Tobacco Induced Diseases, vol. 13, no. 1, article no 38, 2015.

[8] C. Haig, D. Carrick, J. Carberry et al., "Current smoking and prognosis after acute ST-segment elevation myocardial infarction," JACC: Cardiovascular Imaging, 2018.

[9] Task Force Members, "ESC guidelines on the management of stable coronary artery disease: the Task Force on the management of stable coronary artery disease of the European Society of Cardiology," European Heart Journal, vol. 34, no. 38, pp. 2949-3003, 2013.

[10] M. Roffi, C. Patrono, J.-P. Collet et al., "2015 ESC guidelines for the management of acute coronary syndromes in patients presenting without persistent ST-segment elevation. task force for the management of acute coronary syndromes in patients presenting without persistent ST-segment elevation of the European society of cardiology (ESC)," European Heart Journal, vol. 37, no. 3, pp. 267-315, 2016.

[11] S. Windecker, P. Kolh, F. Alfonso et al., "2014 ESC/EACTS guidelines on myocardial revascularization. the task force on myocardial revascularization of the European society of cardiology (ESC) and the European association for cardio-thoracic surgery (EACTS)," European Heart Journal, vol. 31, no. 4, pp. 264-267, 2015.

[12] E. A. Amsterdam, N. K. Wenger, R. G. Brindis et al., "2014 AHA/ACC guideline for the management of patients with non-ST-elevation acute coronary syndromes: a report of the American college of cardiology/American heart association task force on practice guidelines," Journal of the American College of Cardiology, vol. 64, no. 24, pp. e139-e228, 2014.

[13] G. I. Barbash, J. Reiner, H. D. White et al., "Evaluation of paradoxic beneficial effects of smoking in patients receiving thrombolytic therapy for acute myocardial infarction: mechanism of the "smoker's paradox" from the GUSTO-I trial, with angiographic insights. global utilization of streptokinase and tissue-plasminogen activator for occluded coronary arteries," Journal of the American College of Cardiology, vol. 26, no. 5, pp. 1222-1229, 1995.

[14] G. K. Andrikopoulos, D. J. Richter, P. E. Dilaveris et al., "Inhospital mortality of habitual cigarette smokers after acute myocardial infarction; the "smoker's paradox" in a countrywide study," European Heart Journal, vol. 22, no. 9, pp. 776-784, 2001.

[15] D. Hasdai, C. S. Rihal, A. Lerman, D. E. Grill, and D. R. Holmes Jr., "Smokers undergoing percutaneous coronary revascularization present with fewer narrowings in the target coronary artery," American Journal of Cardiology, vol. 80, no. 9, pp. 12121214, 1997.

[16] G. Gennaro, S. J. Brener, B. Redfors et al., "Effect of smoking on infarct size and major adverse cardiac events in patients with large anterior ST-elevation myocardial infarction (from the INFUSE-AMI trial)," American Journal of Cardiology, vol. 118, no. 8, pp. 1097-1104, 2016.

[17] G. Weisz, D. A. Cox, E. Garcia et al., "Impact of smoking status on outcomes of primary coronary intervention for acute myocardial infarction - The smoker's paradox revisited," American Heart Journal, vol. 150, no. 2, pp. 358-364, 2005.

[18] Q. Chen, R. Wu, and H. Pan, "Association of smoking on outcomes of patients underwent coronary stent implantation," Xin Xue Guan Kang Fu Yi Xue Za Zhi, vol. 22, no. 2, pp. 130-132, 2013 (Chinese).

[19] H. Pan, Q. Liu, D. Pan et al., "Association of smoking on outcomes of patients with coronary artery disease who underwent percutaneous coronary intervention," Zhong Guo Lin Chuang Yan Jiu, vol. 24, no. 10, pp. 895-896, 2011 (Chinese).

[20] Z. Zhu, C. Gao, Z. Niu et al., "Association between smoking status at follow-up and clinical outcomes in patients undergoing successful percutaneous coronary intervention," Chin J Cardiol, vol. 37, no. 9, pp. 777-780, 2009 (Chinese).

[21] Task Force Members, "Fourth universal definition of myocardial infarction," European Heart Journal, pp. 1-33, 2018.

[22] Y. Song, Z. Gao, X. Tang et al., "Impact of residual SYNTAX score on clinical outcomes after incomplete revascularisation percutaneous coronary intervention: A large single-centre study," EuroIntervention, vol. 13, no. 10, pp. 1185-1193, 2017.

[23] M. Saad, G. Fuernau, S. Desch et al., “"Smoker's paradox" in patients with cardiogenic shock complicating myocardial infarction - A substudy of the IABP-SHOCK II-trial and registry," International Journal of Cardiology, vol. 222, pp. 775779, 2016.

[24] J. O. Robertson, R. Ebrahimi, A. J. Lansky, R. Mehran, G. W. Stone, and A. M. Lincoff, "Impact of cigarette smoking on extent of coronary artery disease and prognosis of patients with non-ST-segment elevation acute coronary syndromes: An analysis from the ACUITY trial (acute catheterization and urgent intervention triage strategy)," JACC: Cardiovascular Interventions, vol. 7, no. 4, pp. 372-379, 2014.

[25] C. L. Grines, E. J. Topol, W. W. O’Neill et al., “Effect of cigarette smoking on outcome after thrombolytic therapy for myocardial infarction," Circulation, vol. 91, no. 2, pp. 298-303, 1995.

[26] Y.-H. Sun, Y.-J. Yang, W.-D. Pei, Y.-J. Wu, and R.-L. Gao, "Patients with low high-density lipoprotein-cholesterol or smoking are more likely to develop myocardial infarction among subjects with a visible lesion or stenosis in coronary artery," Circulation Journal, vol. 70, no. 12, pp. 1602-1605, 2006.

[27] N. L. Benowitz, "Pharmacologic aspects of cigarette smoking and nicotine addiction," The New England Journal of Medicine, vol. 319, no. 20, pp. 1318-1330, 1988.

[28] H. Bounameaux, M. Griessen, P. Benedet, B. Krahenbuhl, and A. Deom, "Nicotine induced haemodynamic changes during cigarette smoking and nicotine gum chewing: A placebo controlled study in young healthy volunteers," Cardiovascular Research, vol. 22, no. 2, pp. 154-158, 1988.

[29] D. Lucini, F. Bertocchi, A. Malliani, and M. Pagani, "A controlled study of the autonomic changes produced by habitual cigarette smoking in healthy subjects," Cardiovascular Research, vol. 31, no. 4, pp. 633-639, 1996. 
[30] O. N. Niedermaier, M. L. Smith, L. A. Beightol, Z. ZukowskaGrojec, D. S. Goldstein, and D. L. Eckberg, "Influence of cigarette smoking on human autonomic function," Circulation, vol. 88, no. 2, pp. 562-571, 1993.

[31] A. G. Violaris, A. Thury, E. Regar, R. Melkert, and P. W. Serruys, "Influence of a history of smoking on short term (six month) clinical and angiographic outcome after successful coronary angioplasty," Heart, vol. 84, no. 3, pp. 299-306, 2000.

[32] Y.-J. Zhang, J. Iqbal, D. Van Klaveren et al., "Smoking is associated with adverse clinical outcomes in patients undergoing revascularization with PCI or CABG: The SYNTAX trial at 5year follow-up," Journal of the American College of Cardiology, vol. 65, no. 11, pp. 1107-1115, 2015.

[33] J. Ma, X. Wang, M. Gao, Y. Ding, and Y. Guan, "Effect of smoking status on coronary artery disease among Chinese postmenopausal women," Internal and Emergency Medicine, vol. 11, no. 4, pp. 529-535, 2016.

[34] R. Roberts, "Genetics of coronary artery disease: an update," Methodist DeBakey Cardiovascular Journal, vol. 10, no. 1, pp. 712, 2014.

[35] X. Lu, L. Wang, S. Chen et al., "Genome-wide association study in Han Chinese identifies four new susceptibility loci for coronary artery disease," Nature Genetics, vol. 44, no. 8, pp. 890894, 2012. 


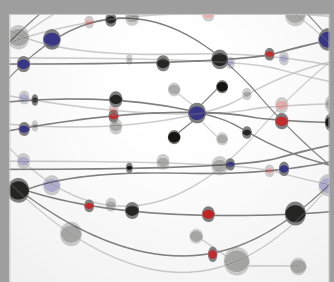

The Scientific World Journal
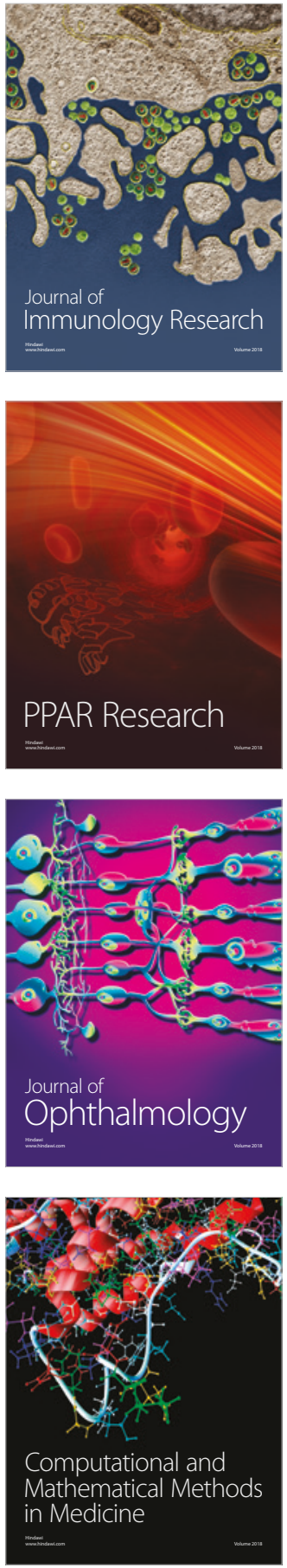

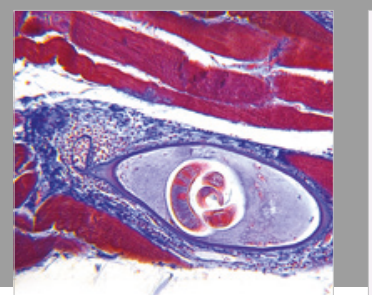

Gastroenterology Research and Practice

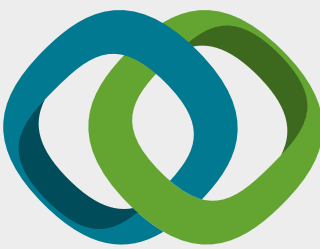

\section{Hindawi}

Submit your manuscripts at

www.hindawi.com
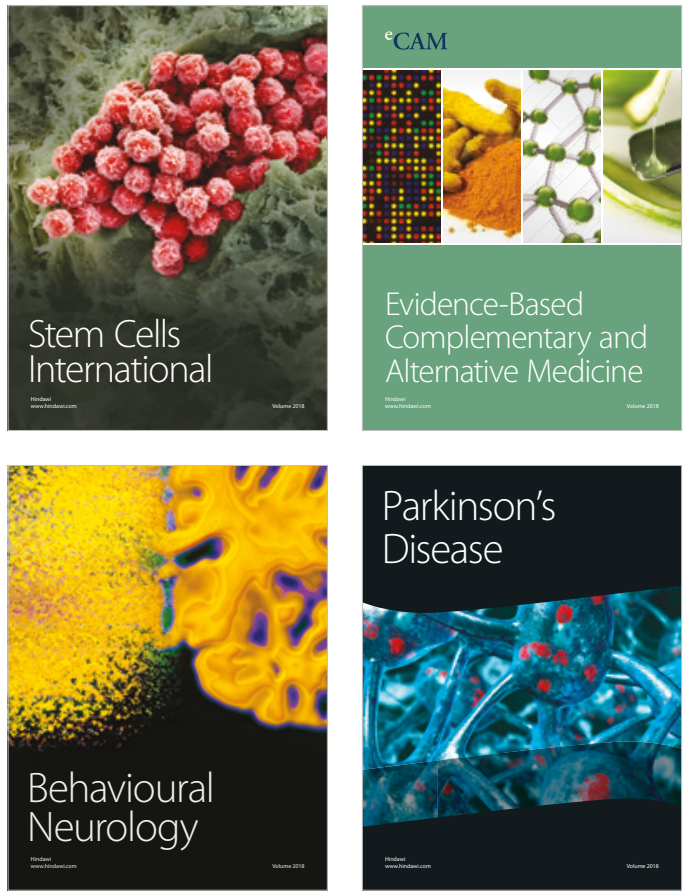

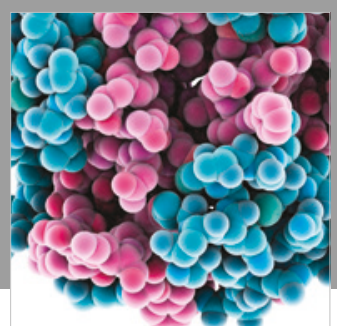

ournal of

Diabetes Research

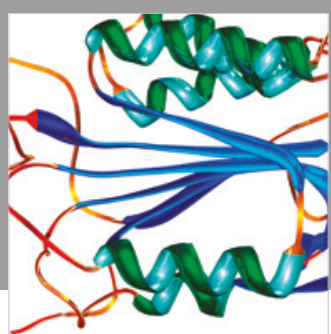

Disease Markers
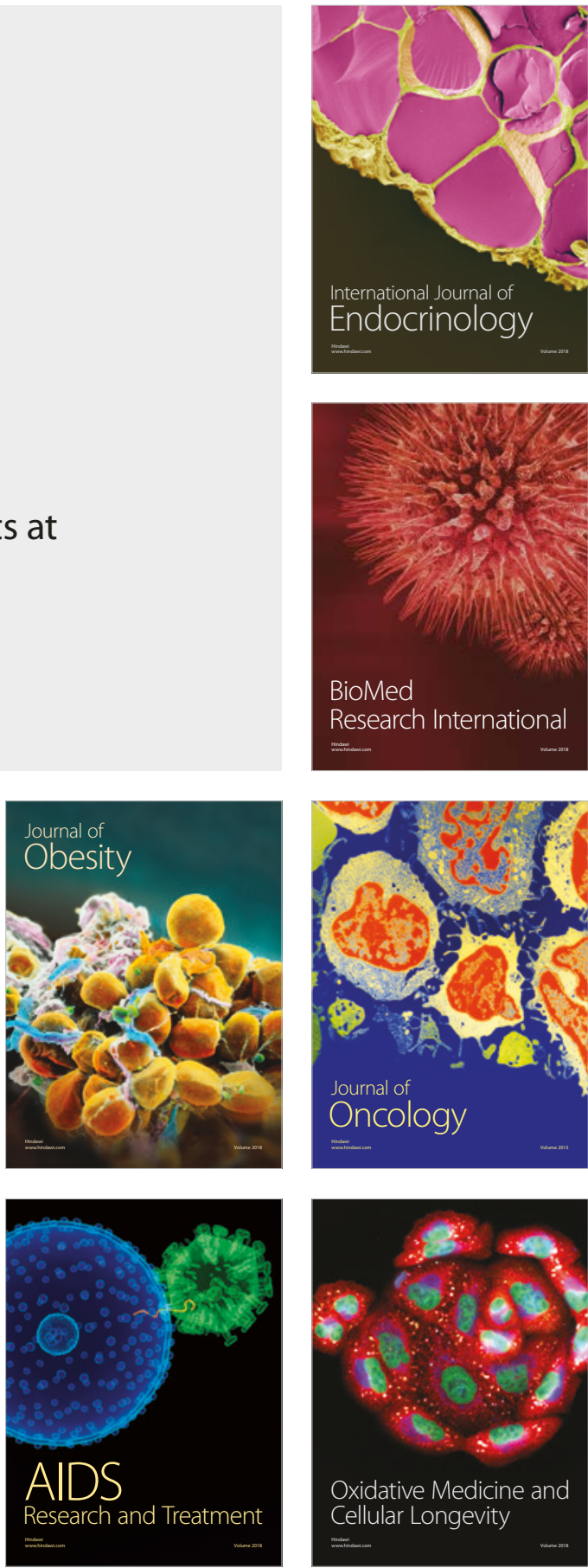